\title{
Role of sagittal anorectoplasty in treating constipation in patients with recto-perineal fistula
}

\author{
Moustafa Mohamed Mahmoud Bayoumi ${ }^{1 *}$ (D, Ayman Mostafa Allam² and Amr Abdelhamid AbouZeid ${ }^{3}$
}

\begin{abstract}
Background: Recto-perineal fistula is a common anorectal anomaly, affecting both males and females. The anus is abnormally anteriorly located and is usually stenotic. Management of recto-perineal fistula is a dialectic problem. The most common presenting complaint is constipation; also, long-term follow-up of these patients revealed high incidence of postoperative constipation.

Results: The study included 30 patients (19 female and 11 male). Their age at time of repair ranged from 3 to 78 months. We compared the pre- and postoperative constipation rate after limited posterior sagittal anorectoplasty (PSARP) using Krickenbeck score. Overall, the rate of constipation has dropped from 77 (23/30) to 30\% (9/30) after surgery. Fourteen patients out of 23 patients with preoperative constipation were cured. Another patient with preoperative constipation improved regarding the grade of constipation. The remaining 8 patients with preoperative constipation did not show improvement after operation. These results were statistically significant (McNemar test). Out of the 9 patients who were still constipated after surgery, 7 had associated fecal soiling that showed good response with bowel management of constipation.
\end{abstract}

Conclusion: Limited posterior sagittal anorectoplasty can have a role in treating constipation among cases of recto-perineal fistula.

Keywords: Anorectal malformation, Constipation, Recto-perineal fistula, Anorectoplasty

\section{Background}

Recto-perineal fistula is a common anorectal anomaly, affecting both males and females [1]. Limited posterior sagittal anorectoplasty (PSARP) is the most common surgical technique used for the repair of recto-perineal fistula [2]. The functional outcomes after the repair of recto-perineal fistula have been considered by some authors as satisfactory. However, long-term follow-up of these patients revealed high incidence of postoperative constipation [3-5]. Not only may constipation persist after surgery, but also some scholars suggested that it may even get worse [6].

In this report, our aim was to study the effect of surgery (limited PSARP) on constipation among patients

\footnotetext{
*Correspondence: mos_bayomi@hotmail.com

${ }^{1}$ Department of Pediatric Surgery, Faculty of Medicine, Ain Shams University,

3 Elhashmy Street, Cairo 13752, Egypt

Full list of author information is available at the end of the article
}

with recto-perineal fistula using Krickenbeck classification for postoperative outcomes.

\section{Methods}

A retrospective study was conducted at two tertiary centers of pediatric surgery between January 2018 and August 2018. The study included all patients with rectoperineal fistula who underwent limited PSARP in the last 5 years. Patients with sacral dysplasia and/or spinal anomalies (tethered cord/meningomyelocele) were excluded. Also, we excluded those who underwent other forms of simpler anoplasties ( $\mathrm{Y}-\mathrm{V}$ anoplasty; cut-back anoplasty; posterior "Hendren" anoplasty) [7]. The study was conducted after approval of the internal review board.

Medical records of patients in the study were reviewed for age, sex, presence of preoperative constipation, type of operation, occurrence of postoperative wound 
dehiscence (partial or complete), and if secondary sutures were taken or covering colostomy was done.

Krickenbeck classification for postoperative outcomes was used to compare the grade of constipation before and after the operation. Constipation is defined as the incapacity to empty the rectum spontaneously every day. Constipation was assessed and graded as shown in (Table 1) [8]. If defecation occurred only by using suppositories or anal dilators, the patient was considered grade 2 constipation. Postoperative soiling was assessed and graded as shown in (Table 1).

The collected data was revised, coded, tabulated, and introduced to the PC (personal computer) using Statistical Package for Social Science SPSS 17 (SPSS Inc., Chicago, IL). Data was presented as percentage as indicated. Comparison between rate of constipation preand postoperatively in patients with recto-perineal fistula was performed using McNemar's test.

\section{Operative technique}

Operations were performed by surgeons with at least a 7 -year experience in pediatric surgery. The same steps of the repair were followed in all cases [9]. A circumferential incision was made around the fistula and extended backwards to open the muscle complex (racket-shaped incision). The incision was deepened till reaching the rectal fascia. Dissection of peri-rectal fascia started posteriorly and laterally followed by the separation of the rectum from the more adherent anterior structures (vagina/urethra) [10-12]. The mobilized anorectum was then placed orthotopically within the muscle complex, followed by reconstruction of the perineal muscles and finally the ano-cutaneous anastomosis was performed (Fig. 1).

In absence of wound disruption, we resume feeding on the 3rd postoperative day. With the progress of oral intake, we introduce laxatives (stool softeners) to overcome pain in the early postoperative period. Laxatives are continued for 4-6 weeks postoperatively. Anal calibration with metal dilators is routine starting 2-3 weeks postoperatively and is continued for $2-4$ months.

Table 1 Assessment and grading of constipation and soiling using Krickenbeck classification in patients with recto-perineal fistula before and after the repair

\begin{tabular}{ll}
\hline Constipation & Yes/no \\
Manageable by changes in diet & Grade 1 \\
Requires laxative & Grade 2 \\
Resistance to diet and laxatives, on enema & Grade 3 \\
Soiling & Yes/no \\
Occasional staining of underwear & Grade 1 \\
Every day without social problem & Grade 2 \\
Constant with social problem & Grade 3 \\
\hline
\end{tabular}

\section{Results}

We reviewed files of 60 cases with recto-perineal fistula who were operated in the last 5 years. We excluded 20 cases who underwent operations other than limited PSARP. Ten cases were lost to follow-up. The study included the remaining 30 cases (19 female and 11 male). Their age at time of repair ranged from 3 to 78 months with a median age of 7.5 months. The duration of follow-up ranged from 4 to 67 months with a mean of 37 months.

As regards constipation, 23 out of 30 patients included in the study were constipated before surgery. Fourteen of these constipated patients were relieved from constipation after surgery, and one patient got improved as regard the constipation grade and frequency. The remaining 8 patients did not show improvement in constipation before and after surgery (Fig. 2). When tested statistically using McNemar's test, there is a significant difference between the rate of constipation before and after surgery (Table 2).

There were seven children who did not have constipation neither before nor after surgical repair. The indication for operation in these patients was abnormal location of the anus (cosmetic reason).

Voluntary bowel control could not be assessed in seven patients who did not reach the age of bowel control during the period of the study; however, the rest of patients had voluntary bowel movements. Postoperative soiling was reported in 7 constipated patients; one of them had frequent staining of underwear (grade 2; Table 1), while the remaining 6 cases had constant soiling with social problems (grade 3). Soiling in all cases showed marked improvement after managing constipation with stimulant laxatives (Senna derivatives).

Wound dehiscence complicated the surgical repair in six patients: four cases had minor wound dehiscence that was treated conservatively in three and one case required secondary sutures, and another two cases had major wound dehiscence necessitating fecal diversion (covering colostomy) and secondary sutures. From all cases with wound dehiscence, only one case suffered from persistent postoperative constipation (same grade as preoperative).

\section{Discussion}

Recto-perineal fistula is a common anorectal anomaly in which the anus is abnormally located in the perineum anterior to the center of the muscle complex. Usually, a part of the anal canal is present within the sphincter mechanism, so most of these patients would be expected to have a good chance for fecal continence after the repair [13]. However, fecal soiling can occur in some of these patients usually associating severe constipation (over retentive stool incontinence; ORSI) [14]. 

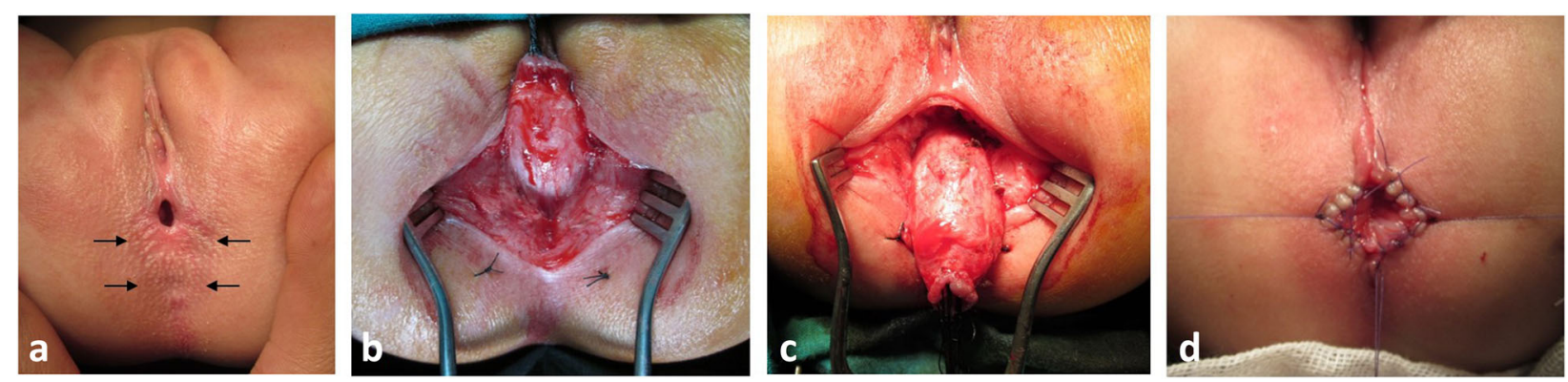

Fig. 1 Thirteen-month-old female with recto-perineal fistula underwent limited sagittal anorectoplasty. a In the supine position, we can see the anus anteriorly displaced (black arrows are pointing to the normal predestined site of the anus). $\mathbf{b}$ Dissection of the anorectum starts on the posterior and lateral aspects. c Separation of the anorectum from the intimately adherent vagina on the anterior aspect. $\mathbf{d}$ Anoplasty performed after repositioning of the anorectum backwards at the predestined site of the anus

Management of recto-perineal fistula is controversial. Some surgeons prefer not to do surgical correction especially when the fistula is sufficiently wide because they assume that there is a high incidence of postoperative constipation that would make surgery of little or no value. Others would prefer prolonged regular dilatation for managing a narrow fistula [15]. Surgical options for recto-perineal fistula include simple anoplasties ( $\mathrm{Y}-\mathrm{V}$ anoplasty; cut-back; posterior anoplasty) and limited posterior sagittal anorectoplasty (PSARP). Hendren described his technique of posterior anoplasty that include incomplete circumferential incision around the anus (from 1 to 11 o'clock position). He reported successful outcome in treating constipation with a relatively lower risk of wound complications [7]. However, simple anoplasties may be suitable only for cases with mild anterior anal displacement $[10,15]$. On the other hand, limited
PSARP can be used to correct all degrees of rectoperineal fistulae by complete mobilization and repositioning of the anorectum backwards within the muscle complex, but this may be associated with a higher risk of wound dehiscence and anal retraction that may necessitate temporary fecal diversion (colostomy) [11, 12].

One reason for this study was to answer the following question: Does limited PSARP have a role in treating or improving constipation in patients with recto-perineal fistula?

Constipation is a common finding after surgical repair of low anorectal anomalies. The reason for constipation is unclear and it seems to occur regardless of the used surgical technique $[3,16]$. Preoperative constipation rate in our study was $77 \%(23 / 30)$ which has been improved after limited SARP to $30 \%(9 / 30)$. This postoperative constipation rate is lower than many reported studies

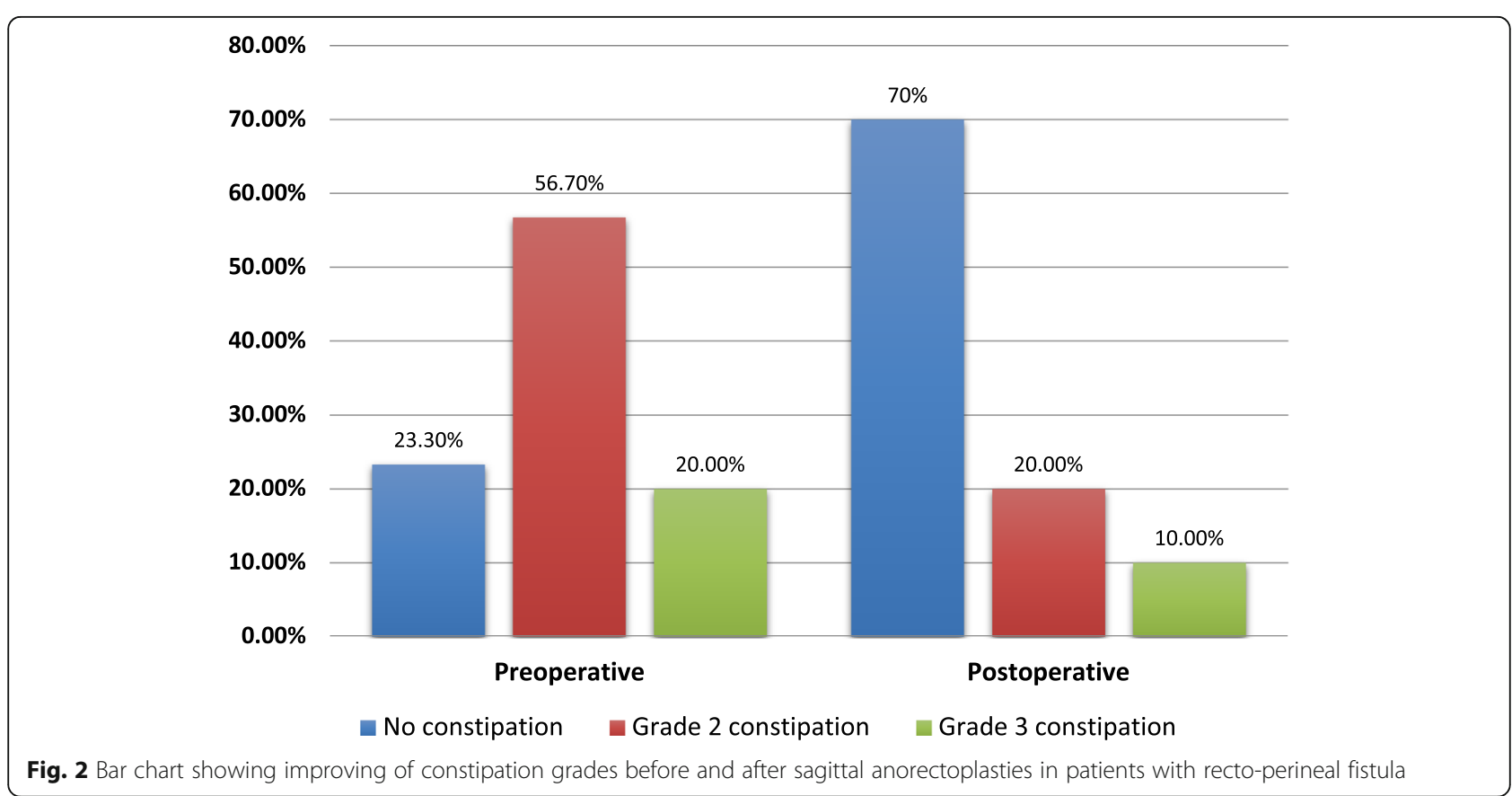


Table 2 Comparison between rate of constipation preoperatively and postoperatively in patients with recto-perineal fistula using McNemar's test

\begin{tabular}{|c|c|c|c|c|c|c|}
\hline & & \multicolumn{2}{|c|}{ Postoperative constipation } & \multirow[t]{2}{*}{ Total } & \multicolumn{2}{|c|}{ McNemar's test } \\
\hline & & No & Yes & & & \\
\hline \multirow[t]{2}{*}{ Preoperative constipation } & No & $7(23.3 \%)$ & 0 & $7(23.3 \%)$ & $p$ value & Sig. \\
\hline & Yes & $14(46.7 \%)$ & $9(30 \%)$ & $23(76.7 \%)$ & 0.0005 & S \\
\hline Total & & $21(70 \%)$ & $9(30 \%)$ & $30(100 \%)$ & & \\
\hline
\end{tabular}

$[3,4,16]$. Constipation before and after the repair was graded according to Krickenbeck classification [8]. Most of the cases who had preoperative constipation $(14 / 23)$ were treated from constipation. One case $(1 / 23)$ has been improved as regard constipation grade (from grade 3 to grade 2$)$. The remaining 8 cases $(8 / 23)$ are still having the same degree of constipation. According to these results, repair of recto-perineal fistula by limited PSARP treat or at least improve constipation in about two third of cases who were suffering from constipation.

We do agree that the severity of constipation can change with time as patients go older, but this is not expected to occur during the early years of life. Kyrklund et al. reported significant constipation among cases of recto-perineal fistula treated by simple anoplasties; however, significant improvement of constipation was noticed at follow-up in older children ( $>12$ years of age) [17]. In our study, most operations were performed in infancy and were followed by improvement of constipation in this young age group that would suggest the effect to be directly related to surgery. Although we cannot exclude a possible "halo" effect of the surgical procedure that may result in some symptomatic improvement; however, this halo effect should be temporary and not expected to persist (median follow-up in our study was 40 months). Another possible external influence may be related to postoperative dietary change. In our study group, weaning from breastfeeding was a common dietary change after operation influenced by the period of perioperative fasting. This would probably aggravate constipation rather than improve it.

From previous studies, we have learned that constipation in anorectal anomalies may be attributed to multiple factors (anterior anal displacement, narrow anus, colonic dysmotility, neurogenic factors) [18]. Among these factors, the abnormal anterior displacement of the anal canal within the pelvic muscles can disturb the normal orientation of the longitudinal axis of anal canal in relation to the surrounding vector forces created by these muscles during defecation [18]. A potential benefit of surgery (limited PSARP) is restoring the normal orientation of the anal canal within the pelvic musculature that can explain improvement of constipation in these cases after operation [7, 19].
Another possible beneficial effect of surgery would be removing of a stenotic termination of the recto-perineal fistula, which we have noticed to be more common in male patients.

Soiling is defined as fecal staining of underwear which occurs because of defects in the sphincteric mechanism or as a consequence of protracted constipation (overflow incontinence) [20, 21]. Previous studies reported soiling rate in low anorectal anomalies ranging from 9 to $48 \%$ $[3,4,16]$. In our study group, seven cases $(23 \%)$ had soiling; all of them were constipated. Treatment of constipation by stimulant laxatives (Senna derivatives) markedly improved soiling in all cases that would suggest the cause of soiling in these patients to be due to overflow (pseudo-incontinence) rather than true incontinence from sphincteric defects.

Wound dehiscence at the mucocutaneous junction after sagittal anorectoplasty is a common complication $[9,22]$, that may lead to delayed functional sequelae (constipation and soiling) [16]. However, in this study we could not find significant correlation between the occurrence of wound dehiscence and delayed functional sequelae. We reported 6 patients $(20 \%)$ with wound dehiscence; 3 cases had minor dehiscence which was managed non-operatively, another case of minor dehiscence was managed by secondary sutures, and 2 cases had major dehiscence that needed covering colostomy and secondary sutures. From all cases with wound dehiscence, only one case had persistent postoperative constipation.

Some scholars question the value of limited PSARP in the repair of recto-perineal fistula and may consider it a sort of overdoing [15], also it raises the concern toward sphincter muscle dissection and possibility of true incontinence which is not found among our patients. To answer this question, it may be more appropriate to compare the repaired cohort with an unrepaired cohort, as well as comparing limited PSARP with other simpler anoplasties. However, in this report, we tried to address this issue from a different perspective by comparing constipation in the same subject before and after the repair. The study still may be criticized for the small sample size and absence of long-term follow-up in all cases which is important to get more representative results about delayed functional sequelae. 


\section{Conclusion}

Limited posterior sagittal anorectoplasty can have a role in treating constipation among cases of recto-perineal fistula, although future comparative randomized studies between different surgical techniques are needed with larger sample of patients and long-term follow-up.

\section{Abbreviations}

PSARP: Posterior sagittal anorectoplasty; ORSI: Over retentive stool

incontinence

\section{Acknowledgements}

Not applicable

\section{Authors' contributions}

All authors contributed to the study conception and design. Material preparation, data collection and analysis were performed by MMB, AMA, and AAA. The first draft of the manuscript was written by MMB and all authors commented on previous versions of the manuscript. All authors read and approved the final manuscript.

\section{Funding}

Not applicable

\section{Availability of data and materials}

The data that support the findings of this study are available on request from the corresponding author MB. The data are not publicly available because they are containing information that could compromise research participant consent.

\section{Ethics approval and consent to participate}

Owing to the retrospective nature of the study, an IRB number was not required, and the study was approved through expedited review by the scientific/ethical committee of the Surgery Department (Faculty of Medicine, Ain-Shams University). All procedures performed in this study were in accordance with the ethical standards of the institutional and national research committee. Informed verbal consent was obtained from all individual participants included in the study.

\section{Consent for publication}

Not applicable

\section{Competing interests}

The authors declare that they have no competing interests.

\section{Author details}

'Department of Pediatric Surgery, Faculty of Medicine, Ain Shams University, 3 Elhashmy Street, Cairo 13752, Egypt. ${ }^{2}$ Department of Pediatric Surgery, Ain Shams University, 12 Elhomossany Street, Kobri Elkoba, Cairo 11766, Egypt. ${ }^{3}$ Department of Pediatric Surgery, Ain Shams University, Lotefy El-Sayed Street, 9 Ain-Shams University buildings, Abbassia, Cairo 11657, Egypt.

Received: 3 October 2019 Accepted: 24 December 2019

Published online: 22 January 2020

\section{References}

1. Pena A, Hong A. Advances in the management of anorectal malformations. Am J Surg. 2000;180(5):370-6.

2. Pena A, Devries PA. Posterior sagittal anorectoplasty: important technical considerations and new applications. J Pediatr Surg. 1982;17:796-811.

3. Rintala $R$, Lindahl $H$, Rasanen M. Do children with repaired low anorectal malformations have normal bowel function? J Pediatr Surg. 1997;32(6):823-6.

4. Pakarinen M, Goyal A, Koivusalo A, et al. Functional outcome in correction of perineal fistula in boys with anoplasty versus posterior sagittal anorectoplasty. Pediatr Surg Int. 2006;22(12):961-5.

5. Hassett S, Snell S, Hughes-Thomas A, et al. 10-Year outcome of children born with anorectal malformation, treated by posterior sagittal anorectoplasty, assessed according to the Krickenbeck classification. J Pediatr Surg. 2009;44(2):399-403.
6. Pena A, Bischoff A, eds. Rectoperineal fistula. Surgical treatment of anorectal problems in children. Springer (eBook); 2015. pp. 107-127.

7. Hendren $\mathrm{WH}$. Constipation caused by anterior location of the anus and its surgical correction. J Pediatr Surg. 1978;13:505-12.

8. Holschneider A, Hutson J, Pena A, et al. Preliminary report on the International Conference for the Development of Standards for the Treatment of Anorectal Malformations. J Pediatr Surg. 2005;40(10):1521-6.

9. AbouZeid AA. Securing the mucocutaneous anastomosis in the repair of low-anorectal anomalies. Ann Pediatr Surg. 2015;11(3):185-91.

10. Javid PJ, Barnhart DC, Hirschl RB, et al. Immediate and long-term results of surgical management of low imperforate anus in girls. J Pediatr Surg. 1998;33:198-203.

11. Kumar B, Kandpal DK, Sharma SB, et al. Single-stage repair of vestibular and perineal fistulae without colostomy. J Pediatr Surg. 2008;43:1848-52.

12. Kuijper CF, Aronson DC. Anterior or posterior sagittal anorectoplasty without colostomy for low-type anorectal malformation: how to get a better outcome? J Pediatr Surg. 2010;45:1505-8.

13. Levitt MA, Pena A. Operative management of anomalies in males. In: Holschneider AM, Hutson JM, editors. Anorectal malformations in children. Berlin Heidelberg: Springer-Verlag; 2006. p. 295-302.

14. Pena A, Levitt MA. Colonic inertia disorders in pediatrics. Curr Probl Surg. 2002:39:666-730

15. Pakarinen MP, Rintala RJ. Management and outcome of low anorectal malformations. Pediatr Surg Int. 2010:26:1057-63.

16. Levitt MA, Pena A. Review anorectal malformations. Orphanet J Rare Dis. 2007;2:33-6.

17. Kyrklund K, Pakarinen MP, Taskinen S, Rintala RJ. Bowel function and lower urinary tract symptoms in males with low anorectal malformations: an update of controlled, long-term outcomes. Int J Color Dis. 2015;30:221-8.

18. AbouZeid AA, Radwan AB, Mohammad SA, et al. Suboptimal correction of low anorectal anomalies: a possible cause for intractable constipation in children. Ann Pediatr Surg. 2016;12:142-9.

19. AbouZeid AA, Mohammad SA, Khairy KT. MRI anatomy of anteriorly displaced anus: what obstructs defecation? Pediatr Radiol. 2014;44:831-8.

20. Yeung CK, Kiely EM. Low anorectal anomalies: a critical appraisal. Pediatr Surg Int. 1991;6:333-5.

21. Cheu HW, Grosfeld JL. The atonic baggy rectum: a cause of intractable obstipation after imperforate anus repair. J Pediatr Surg. 1992;27:1071-3.

22. Allam A, Abou Zeid A, El Shafei I, et al. Repair of low anorectal anomalies in female patients. Annals of Pediatric Surgery. 2017;13(3):140-4.

\section{Publisher's Note}

Springer Nature remains neutral with regard to jurisdictional claims in published maps and institutional affiliations.

\section{Submit your manuscript to a SpringerOpen ${ }^{\circ}$ journal and benefit from:}

- Convenient online submission

- Rigorous peer review

- Open access: articles freely available online

High visibility within the field

- Retaining the copyright to your article

Submit your next manuscript at $>$ springeropen.com 